\title{
Fenomenología
}

\section{La influencia de la cultura occidental en los cuidados del cuerpo relativos a la estética, a la actividad física y a la alimentación}

\section{The influence of western culture in the care of the body relative to aesthetics, physical activity and food}

\section{A influência da cultura ocidental no cuidado do corpo em relação à estética, atividade física e alimentação \\ Celia Beltrán Beltrán. \\ Graduada en Enfermeria, Universitat de València.}

Cómo citar este artículo en edición digital: Beltrán Beltrán, C. (2012) La influencia de la cultura occidental en los cuidados del cuerpo relativos a la estética, a la actividad física y a la alimentación.16, 34. Disponible en: http://dx.doi.org/10.7184/ cuid.2012.34.02>

Correspondencia: Avenida Blasco Ibáñez, 13; 46010, Valencia, Spain.e-mail: celiabeltrandue@gmail.com Recibido 20/03/2012/ Aceptado: 17/06/2012

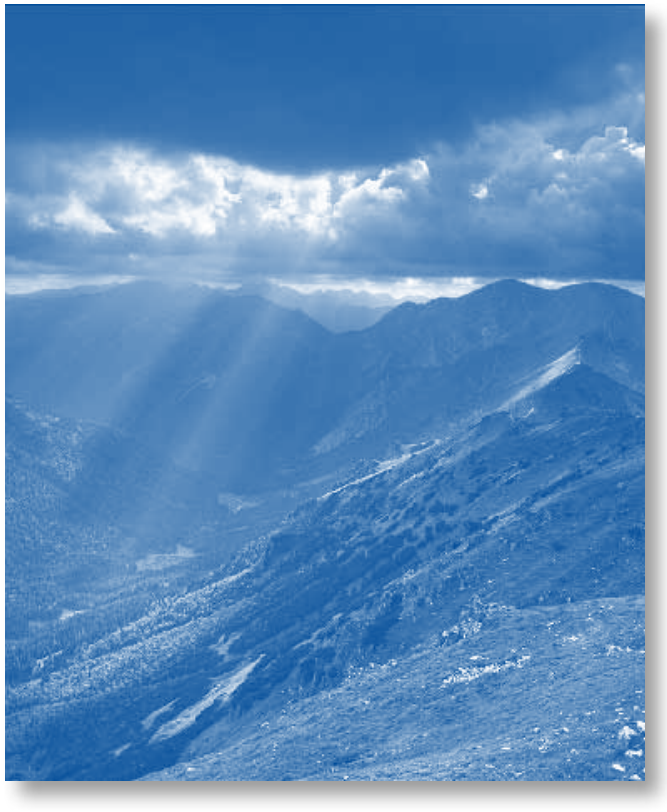

\section{ABSTRACT}

The body is part of the personal identity of each individual, and as identity does, it changes along lifetime, so it is perceived differently in each life phase and it is used by the indi- vidual to express their own identity. The body is something more than a set of differentiated cells and it is highly valued in every society. Thus, different patterns of body care represent each culture. In this sense, we are asking how western culture is conditioning body care behaviors relative to aesthetics, physical activity and alimentation. These care behaviors vary among populations according to the values that they associate to body image. In the same way, they also differ within each population, as these values evolve over time. Ultimately, culture is generating a series of behaviors that can become risk factors for some diseases. Thus, nurses will have to identify those potentially harmful body care behaviors to encourage alternative healthy behaviors, focusing on evaluations, availability, motivations, rewards, preferences, aversions and self-concept of each individual in each age group.

Key words: Culture: Body Image, Body Care, Prevention, Nursing. 


\section{RESUMO}

O corpo forma parte da identidade pessoal de cada individuo, ao igual que ela muda ao longo da vida por o que em cada etapa percebe-se de diferente maneira e com ele expressa-se a propia identidade. O corpo é algo mais que um conjunto de células diferenciadas e em todas as sociedades tem-se em alta estima pelos cuidados corporais representam a cada cultura. Portanto, nos representamos como a cultura occidental condiciona os cuidados do corpo relativos à estética, a actividade física e a alimentação. Ditos cuidados variam entre as povoações segundo os valores que nelas se associação com a imagem corporal, da mesma maneira, também variam dentro da mesma povoação, pois estes valores evolucionam com o passar dos anos. Em definitiva a cultura está gerando uma serie de condutas que podem chegar a constituir factores de risco de algumas doenças. De maneira que o pessoal de enfermaria identificará os cuidados corporais prejudiciais para promover condutas saudáveis atendendo as valorizações, a disponibilidade, as motivações, os esforços, as preferências, as adversões e auto-conceito de cada individuo em cada grupo de idade.

Palavras chave: Cultura, Imagem Corporal, Cuidados Corporais, Prevenção, Enfermaria.

\section{RESUMEN}

El cuerpo forma parte de la identidad personal de cada individuo, al igual que ella cambia a lo largo de la vida por lo que en cada etapa se percibe de diferente manera y con él se expresa la propia identidad. El cuerpo es algo más que un conjunto de células diferenciadas y en todas las sociedades se tiene en alta estima por lo que los cuidados corporales representan a cada cultura. Por lo tanto, nos planteamos cómo la cultura occidental condiciona los cui- dados del cuerpo relativos a la estética, la actividad física y la alimentación. Dichos cuidados varían entre las poblaciones según los valores que en ellas se asocian con la imagen corporal, de la misma manera, también varían dentro de la misma población, pues estos valores evolucionan con el paso de los años. En definitiva la cultura está generando una serie de conductas que pueden llegar a constituir factores de riesgo de algunas enfermedades. De manera que el personal de enfermería identificará los cuidados corporales perjudiciales para fomentar conductas saludables atendiendo a las valoraciones, la disponibilidad, las motivaciones, los refuerzos, las preferencias, las aversiones y el autoconcepto de cada individuo en cada grupo de edad.

Palabras clave: Imagen Corporal, Cuidados Corporales, Prevención, Enfermería.

\section{INTRODUCCIÓN}

El cuerpo es un vehículo de expresión de la personalidad, por ello se busca alcanzar una imagen corporal representativa. Hoy en día, la imagen corporal es muy valorada en nuestra sociedad por lo que los cuidados corporales tienen especial importancia para todos los individuos en todas las etapas de la vida. Concretamente los cuidados corporales relativos a la estética, la actividad física y la alimentación representan una forma de vida y son la inmediata expresión de la personalidad, un conjunto de rutinas que pueden ser más o menos visibles, de manera que los cuidados corporales, la salud, la vida sana -sin tabaco, naturalmente-, la alimentación equilibrada, el aeróbic, la musculación, la moda vestimentaria, la belleza, la cirugía estética y un largo etcétera de prácticas- y de inversiones económicas- referidas al cuerpo se nos proponen como las técnicas más 
adecuadas para lograr que éste exprese nuestra auténtica identidad como seres únicos, irrepetibles (Méndez, 2002).

Generalmente son repetitivos y se convierten en rutinas hasta finalmente constituir un estilo de vida. Los cuales pueden ser más o menos saludables, ya que dependiendo del contexto social y personal pueden facilitar, o no, el desarrollobiopsicosocial. Como decíamos, existen muchos y variados cuidados del cuerpo, pero aunque algunos sean más visibles que otros, todos son apreciables, pues repercuten en la apariencia física, en la psicología individual y en las relaciones sociales y pueden representar factores de riesgo para determinadas enfermedades. Además, hay que resaltar, que en cada cultura, están condicionados por los valores de la misma. Ello se refleja claramente en los roles de género porque la sociedad espera que el género masculino se comporte de un modo, mientras que el género femenino, de otro distinto.

Esos comportamientos, forman parte de la identidad de manera que los cuidados corporales también serán diferentes en cada género ya que cada grupo concibe y valora su propio cuerpo de diferente forma.

\section{DESARROLLO}

Con todo ello, podemos adivinar que existe una gran diversidad dentro del mundo de los cuidados corporales. Entre los cuales destacan los que se engloban en la estética corporal, en la actividad física y en la alimentación. Concretamente la importancia de la estética ha estado creciendo los últimos años, tanto en hombres como en mujeres y ello se ha extendido a todas las edades.

Dentro del contexto occidental, la estética en la adolescencia juega un papel importante porque es una forma de expresión personal ya

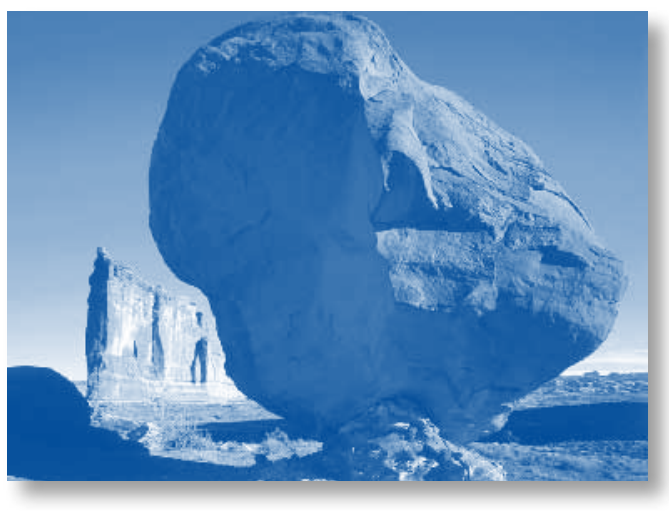

que en: "el contexto de las culturas juveniles urbanas, las prácticas del tatuaje y en general de las perforaciones corporales, se nutren también de las construcciones sociales que las diferentes épocas realizan sobre los cuerpos" (Ganter, 2006). En éste periodo de transición hacia la edad adulta, el cuerpo les ayuda a encontrar su identidad personal así como su identidad grupal, pues la pertenencia a un grupo les aporta seguridad en un mundo de cambios.

Un modo de sentimiento de pertenencia a un grupo es mediante similitudes en sus cuidados corporales, por lo que los cuidados estéticos entre los miembros de un mismo grupo de amistades serán parecidos. Es decir, las tendencias acerca de la imagen corporal, como por ejemplo el corte de pelo, las perforaciones corporales, el uso de cosméticos, los tintes del pelo, el bronceado de la piel,... y sus respectivos cuidados o ritos para conseguir una determinada apariencia, valorada y aceptada por el grupo, serán similares.

Sin embargo, con la incorporación al mercado laboral, muchos cuidados cambian, pues la apariencia del cuerpo se percibe de otra manera, y como consecuencia, los cuidados estéticos cambian. Ello no significa que la imagen corporal deja de ser importante, pues ésta preocupa en todas las edades y a ambos sexos. Sin embargo, durante mucho tiempo en 
la sociedad occidental en la que vivimos se ha adjudicado exclusivamente a las mujeres el estereotipo según el cual el cuidado corporal es sinónimo de belleza. Pero ya va quedando en desuso, pues actualmente se relaciona también con los hombres. La realidad es que cada vez existen más cosméticos para sus cuidados diarios después de afeitado, el contorno de ojos para disimular la apariencia cansada, depilaciones corporales e incluso cirugía estética.

Por lo que respecta a la vejez, nos cuesta asociarlo con estética, ya que automáticamente relacionamos este periodo con el deterioro físico, mental y social (Sarabia, 2012). Sin embargo, en el mundo occidental nos resulta muy común el uso de prótesis dentales en toda la población anciana. "Para el paciente anciano la prótesis dental es una integradora familiar, social, mantenedora de la salud general y elevadora de la expectativa de vida" (De Almeida, 2007) ya que mejora su imagen corporal y por tanto su autoconcepto y autoestima además de mejorar una actividad fisiológica muy necesaria como es comer. Al igual que en las etapas anteriores, en la vejez siguen buscando una imagen corporal que sea aceptada y valorada por el grupo al que pertenecen. Es decir, la apariencia sigue siendo una forma de expresión de la personalidad y está condicionada por los valores que se le adjudican al cuerpo.

Como ya habíamos dicho, además de los cuidados estéticos que son fácilmente visibles (peinados, cirugía estética, prótesis dentales) también hay otros que a la larga se aprecian y constituyen factores de riesgo de algunas enfermedades. Éstos cuidados se reflejan en el estilo de vida y son los relacionados con la actividad física y con la alimentación.

Respecto al ejercicio físico en los países occidentales, generalmente se priorizan las circunstancias personales como el trabajo o los estudios en determinados momentos por encima de la actividad física. También influyen en esta sociedad la facilidad de acceso a los recursos materiales y un fuerte entorno social donde están presentes los hábitos de vida saludables para realizar una mayor actividad física o deportiva. Además, existe una clara $<<$ relación del autoconcepto físico con diferentes variables sociodemograficas como la edad, el género y la práctica físico-deportiva extraescolar > (Moreno, 2008). La autoestima alta es un factor que predispone a la vida activa respecto al ejercicio, el cual se ve reforzado por dicha actividad física así como por las interacciones sociales que le permite desarrollar. Sin embargo, el factor más importante es la motivación, ya que si la persona carece de ella, difícilmente se iniciaran cuidados de la actividad física y mucho más complicado será mantenerlos en el tiempo.

Dentro de la sociedad occidental, en edades tempranas, se asocia el ejercicio físico con la diversión y es su método para entablar relaciones sociales, es decir, los niños lo perciben como un juego que les permite hacer amigos $y$ pasárselo bien. Por ello, es fácil inculcar éstos hábitos deportivos en la infancia, pues parten de su propia iniciativa. Sin embargo, durante la adolescencia prima la competición pues quieren destacar y ser realmente buenos en algún deporte, así mismo, el refuerzo de las amistades es importante por la relevancia que tienen las relaciones sociales en esta etapa de la vida. Los universitarios que eligen hacer alguna actividad física se deciden por aquello que les aporta mayor diversión. La motivación cambia totalmente en la edad adulta en la que de alguna manera se obligan a realizar ejercicio de forma regular, pues lo empiezan a relacionar con la salud. Ello es totalmente evidente en la vejez, quienes mayoritariamente con- 
ciben el ejercicio como una parte más de su mantenimiento físico pues se manifiestan los beneficios relacionados con la salud, además de favorecer sus redes sociales. (Garita, 2006).

La adolescencia es una etapa difícil en la que debido a los cambios físicos, psicológicos y sociales que en ella se producen, existe una mayor facilidad para adoptar hábitos de vida perjudiciales. Por lo general, tal como señala Castillo (2007): “Aquellos jóvenes físicamente activos consumen menos tabaco y menos alcohol, desayunan con mayor frecuencia y poseen hábitos de alimentación más saludables". Ahora bien, la motivación para hacer ejercicio durante la adolescencia cambia con el género. Así pues, cabe destacar que los chicos hacen ejercicio con fines lúdicos, recreativos y sociales principalmente, mientras que las chicas inician ésta actividad impulsadas por la mejora de su imagen personal y de su salud. Con lo cual, es importante vincular a los adolescentes con el ejercicio para favorecer ciertas actitudes frente a factores de riesgo de futuras enfermedades. Otro factor a considerar es la falta de tiempo debido a las exigencias académicas, por lo que se produce un evidente "Cambio estacional: el número de horas de actividad física aumenta durante los meses de vacaciones" (Blasco, 1996).

De los factores anteriormente mencionados como la disponibilidad, la motivación y el autoconcepto, en la edad adulta éste último adquiere especial relevancia pues si tienen en consideración factores personales como la habilidad para practicar un u otro deporte, la condición física más o menos adecuada para un deporte según sus exigencias, la fuerza o resistencia haciendo actividad física y el autoconcepto físico general para desarrollar una actividad física determinada. Pues como reflejó Guillermo Infante en un artículo: “La mayor cantidad de actividad físico-deportiva corresponde una mejor autopercepción del sujeto en cada uno de estos cuatro componentes del autoconcepto físico" (Infante, 2009) de manera que ambos se condicionan: las personas activas tienen un autoconcepto superior a las sedentarias. De ellos depende en esta etapa su motivación para iniciarse en el deporte, por lo que los hombres practican más ejercicio al tener el autoconcepto más vinculado con la actividad. Esto ha sido producido por una actitud enraizada por la sociedad.

A pesar de que la actividad física, tal como señala Paglilla (2001) "Demora el envejecimiento y aumenta el periodo productivo de la vida"; no es lo más común encontrar a personas de la tercera edad practicando actividad física. Aunque en el periodo de jubilación pueden gozar de tener todavía buena salud y que tienen una gran disponibilidad de tiempo, en esta sociedad no se motiva a los ancianos para hacer deporte. Sin embargo, mejorarían sus condiciones físicas, psiquícas y sociales. Pues la práctica de deporte retrasa el envejecimiento tanto físico como psíquico y enriquece la sociabilidad de las personas. No obstante, en los países occidentales, la tercera edad contrasta con las anteriores etapas porque son las mujeres las que incorporan el ejercicio físico a su estilo de vida. Ello se debe a su mayor facilidad para adaptarse a los cambios que se van produciendo y a su entorno. Martínez (2009) ha estudiado el fenómeno por el cual: "Las mujeres parecen preferir en mayor medida que los varones una práctica de actividad física no deportiva y en espacio cerrado y cubierto, más que en espacio abierto y público"; por lo que en la medida de sus posibilidades personales y sociales, aumentan el ejercicio con el fin de conseguir beneficios para su salud.

Los cuidados respecto a la actividad física 
que cada persona realiza a lo largo de su vida se puede decir que varían en función de su disponibilidad, de su autoconcepto y de sus motivaciones. Por los beneficios que representa el realizar actividad física en cualquier etapa de la vida, hay que considerar las circunstancias personales y sociales de cada grupo para inculcarles dicha actividad y los valores que de ella se derivan.

En cuanto a la alimentación, sabemos que en cualquier contexto cultural es algo más que la nutrición para la supervivencia. Pues todos los cuidados que las personas realizan y están relacionados con la alimentación se vinculan, consciente o inconscientemente, con diferentes creencias o tareas estipuladas a lo largo de la evolución histórica de la sociedad. Por lo que cabe recordar que alimentar, tal como señala Gracia (2009) es algo más que sustentar: "Alimentar es intercambiar, nutrir, cuidar, comunicar".

La alimentación está estrechamente vinculada con la formación de las relaciones personales porque es, principalmente, en eventos sociales que giran alrededor de comidas (a veces, sin importar su calidad) donde surgen dichos nexos. Además, para mantener esos vínculos y en definitiva sociabilizarse, las reuniones que se acuerdan para ello se suelen diseñar en base a un momento del día en el que hay que comer. Puesto que comer, supone un paréntesis en la rutina diaria y como tal, representa un tiempo de comunicación, de distracción, de solicitar consejo. De manera que las relaciones humanas se fortalecen y evolucionan en torno a la alimentación.

Además contribuye a la jerarquización de los roles dentro de la familia, así pues, a la mujer se le asigna el rol de madre. Como tal, en un principio, la sociedad solo esperaba de ella que fuera capaz de preparar comidas muy ela- boradas pero con poco dinero, como muestra continua del rol de madre que desempeña bajo las capacidades de entrega y de sacrificio por su familia, anteponiéndola a sí misma. Es por todo ello que la alimentación contribuye a la separación de géneros y condiciona los cuidados que cada uno ejercen respecto a la misma. Sin embargo, con la evolución de la sociedad, también ha cambiado el rol de madre, pues ahora se espera que trabaje fuera de casa además de hacer las actividades domésticas, aunque modificadas. Por ejemplo, con este nuevo rol es socialmente aceptable que se recurra en algunos casos a la comida preparada. De tal manera que, actualmente madre representa a una mujer empresaria capaz de administrar y gestionar su domicilio sin delegar tareas. Con lo cual, el cuidado familiar sigue estando más vinculado a la mujer (Martín, 2004). Sin embargo, el rol de padre respecto a la alimentación, se relaciona con otras tareas que también conforman esta actividad. Para Arés (1996), el rol masculino consiste en: "Saber, poder y tener para ser proveedor, protector, sostenedor de la familia"; por lo que se le relaciona con trabajos remunerados fuera de casa para conseguir dinero y mantener a la familia. Sin embargo, actualmente, ambos roles se entremezclan en nuestro contexto social. Cuando hablamos de género pensamos en las diferencias biológicas, sin embargo, ésta palabra engloba mucho más que eso, pues existen condicionantes sociales y culturales (que) se relacionan con las oportunidades de la mujer (y del hombre) de acceder a la educación, la alimentación y los servicios de salud. Los comportamientos y modos de vida de cada uno de los sexos relacionados con la salud, también son elementos centrales de la teoría de género (Pérez, 2007).

Estos condicionantes marcan las etapas de la vida en ambos géneros $y$, por supuesto, la 
alimentación también varía. En el mundo occidental, se ha visto reflejado en algunas encuestas que durante la crianza de niños y niñas no se hacen distinciones en la manera de alimentarlos. Pero según parece, en algunas etapas de vida adulta, posiblemente en las no relacionadas con la reproducción, hay una disminución de los cuidados en la alimentación de las mujeres (Pérez, 2007). Esto se debe a que cuando se la relaciona con la reproducción, los cuidados en su alimentación se ponen en alza, pero cuando esta relación se extingue, la mujer prioriza el cuidado de su familia sobre el suyo y el desequilibrio nutricional, sobretodo el sobrepeso en nuestra sociedad destaca más en mujeres que en hombres. Ellos, sin embargo no sufren alteraciones tan marcadas en las diferentes etapas de sus vidas. No obstante, en la etapa estudiantil (especialmente en la universitaria) se adoptan hábitos de vida no saludables y constituyen la base para adoptar a largo plazo conductas que suponen factores de riesgo de enfermedades crónicas no transmisibles (diabetes, HTA...). Entre estos malos hábitos destacan el sedentarismo y el consumo de dietas desequilibradas (comer entre horas, dietas milagro,...), los cuales surgen en ambos sexos pero son más acentuados en mujeres. La causa la atribuyen a la falta de tiempo de forma generalizada (Norman, 2007).

Sabemos pues, que muchas conductas están enraizadas en los roles de género que hombres y mujeres adoptan, y además, están apoyadas por la sociedad de la que forman parte. Por lo que, des del punto de vista de la enfermería es difícil cambiar los cuidados relativos a la estética, a la actividad física y a la alimentación que sean perjudiciales para la salud. A pesar de ello, se debe promover los hábitos saludables en todas las etapas de la vida. Pero hay que destacar, que el punto de partida será el respeto hacia el contexto personal y social de hombres y mujeres, por lo que mediante la negociación se tratará de llegar a pactos en los que la salud biopsicosocial del paciente resulte beneficiada.

Con todo ello, nos encontramos ante diagnósticos enfermeros como disposición para mejorar el autoconcepto (00167), por lo que desde la enfermería se debería mejorar la autoconfianza y la autoestima. Ello lo conseguiríamos explorando las razones para la autocrítica, animando a aceptar nuevos desafío, aumentando sistemas de apoyo y, en definitiva, facilitando el ambiente y las actividades que aumenten la autoestima englobando también los cuidados estéticos. Respecto al posible diagnóstico de sedentarismo (00168) enfermería fomentará el ejercicio determinando la motivación y explorando las experiencias anteriores y las barreras para iniciar una actividad. Además, siempre animará a iniciar o continuar con la actividad física ayudando a desarrollar un programa de ejercicio adecuado al mismo tiempo que ayuda a incorporarlo en la rutina semanal. También podríamos encontrar diagnósticos de desequilibrio nutricional por exceso o por déficit $(00001,00002)$ por lo que les asesoraríamos nutricionalmente desde el respeto identificando conductas que se deseen cambiar y estableciendo metas realistas teniendo en cuenta sus gustos i aversiones (NANDA Internacional, 2009-2011: 181, 130, $75,76)$ (Clasificación de Intervenciones de Enfermería, 2009).

Además, hay que tener en cuenta que actualmente se está dando la evidencia de dos fenómenos conjuntamente que comparten la misma motivación. Éste punto de partida es la preocupación por el peso y la realización de dietas específicas y de ejercicio físico intenso. Por una parte encontramos: "Un aumento en 
el número de mujeres que realizan actividad física intensa y deporte de competición, (y por otra parte) un incremento en la incidencia de alteraciones del comportamiento alimentario" (Heras, 2004).

\section{CONCLUSIONES}

De manera que es especialmente importante enseñar buenos hábitos relacionados con la alimentación y el deporte para tratar de prevenir enfermedades como la anorexia.

Todos los cuidados deben llevarse por igual, es decir, sin detrimento de los demás porque cuando el deseo de una imagen corporal perfecta se convierte en una obsesión, la vida gira en torno a ello y se prima la belleza de la apariencia física por encima del resto de campos. Lo que provoca que estos últimos se desarrollan más deficientemente y a largo plazo, eso deteriora la vida. Un claro ejemplo de ello es una enfermedad conocida como anorexia. Desde enfermería se fomentaran los buenos hábitos de vida y se promocionará la imagen corporal fomentando la autoestima desde una perspectiva socioantropológica. Es decir, contemplando al individuo en su contexto como un ser biopsicosocial y todo ello mediante la negociación. Así se tratará de llegar a acuerdos respecto a las actitudes o a las conductas pero respetando siempre las creencias y los valores.

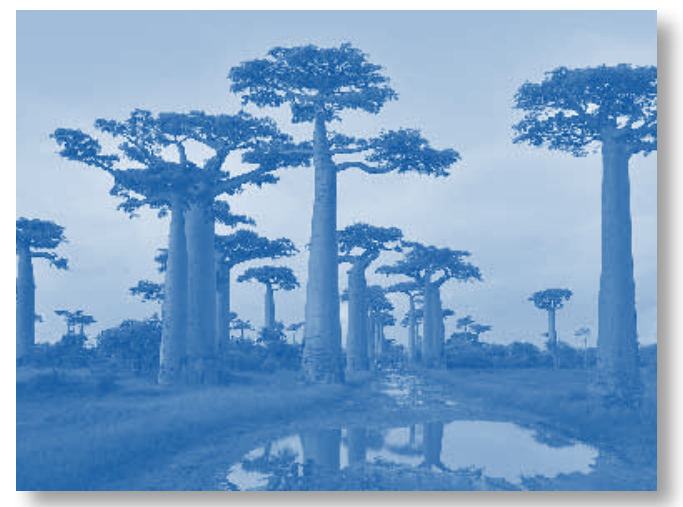

\section{BIBLIOGRAFÍA}

- Arés, P. (1996) Virilidad ¿conocemos el costo de ser hombre? Rev. cuba. psicol. v.13 n.2-3 La Habana 1996. Available: http://pepsic.bvsalud.org/scielo.php?pid=S025743221996000200003\&script=sci_arttext Downloaded 15th May 2012.

- Blasco, T. y otros (1996) Evolución de los patrones de actividad física en estudiantes universitarios. Revista de psicología del deporte. 51-63. Available: http://ddd.uab. cat/pub/revpsidep/19885636v5n2p51.pdf Downloaded 8th May 2012.

- Bulechek, G. (2009) Clasificación de Intervenciones de Enfermería. Elsevier, Madrid.

- Castillo, I. el al. (2007) Efecto de la práctica de actividad física y de la participación deportiva sobre el estilo de vida saludable en la adolescencia en función del género.

- Revista de psicología del deporte, 6 (2), 201-210. Available: http://ddd.uab.cat/pub/revpsidep/19885636v16n2p201. pdf Downloaded 15th May 2012.

- De Almeida, E. et al. (2007) Prótesis dental en el paciente anciano: aspectos relevantes. Rev. Estomatol. Herediana, 17 (2),104-107. Available: http://revistas.concytec.gob.pe/ scielo.php?pid=S1019-43552007000200010\&script=sci_ arttext Downloaded 17th May 2012.

- Ganter, R. (2006) De cuerpos, tatuajes y culturas juveniles. Asociación Venezolana de Sociología. Espacio abierto. 14(1), 25-51. Available: http://www2.scielo.org.ve/pdf/ea/ v15n1-2/art22.pdf Downloaded 17th May 2012.

- Garita, E. (2006) Motivos de participación y satisfacción en la actividad física, el ejercicio físico y el deporte. Revista MHSalud. 3 (1). Available: http://redalyc.uaemex.mx/ redalyc/pdf/2370/237017528002.pdf Downloaded 10th May 2012.

- Gracia, M. (2009) ¿Qué hay hoy para comer?: alimentación cotidiana, trabajo doméstico y relaciones de género. Caderno Espaço feminino. 1(21). Instituto de Estudios de género. Available: http://www.seer.ufu.br/index.php/neguem/article/view/3695 Downloaded 8th May 2012.

- Heather, T. (2010) Diagnósticos enfermeros. NANDA Internacional Elsevier, Madrid. Heras, E. (2004) Alteración de la percepción de la imagen corporal en el deporte. 
- Monografías de psiquiatría, 16(2), 32-40. Available: http:// dialnet.unirioja.es/servlet/articulo? codigo $=1148349$ Fecha consulta: $10 / 5 / 2012$.

- Infante, G. y Goñi, E. (2009) Actividad físico-deportiva y autoconcepto físico en la edad adulta. Revista de psicodidáctica. 14(1), 49-62. Available: http://redalyc.uaemex. $\mathrm{mx} /$ redalyc/pdf/175/17512723004.pdf Downloaded 15th May 2012.

- Martín, E. (2004) El valor de la buena madre. Oficio de ama de casa, alimentación y salud entre las mujeres de clases populares. Revista Española de Sociología. 4. Universidad de Sevilla. Available: http://www.fesweb. org/ publicaciones/res/archivos/res04/04.pdf Downloaded 8th May 2012.

- Martínez, J. et al. (2009) Los hábitos de actividad física de las mujeres mayores en España. Revista Internacionas de Ciencias del Deporte. 5(14). Available: http://www.cafyd. com/REVISTA/01407.pdf Downloaded 10th May 2012.

- Méndez, L. (2002) Cuerpo e identidad. Modelos sexuales, modelos estéticos, modelos identitarios. Pensando el cuerpo, pensando desde del cuerpo. 123-138 Universidad de Castilla-La Mancha Available: http://dcsh.xoc.uam. $\mathrm{mx} /$ sociales/Documentos/Cuerpo_identidad-Modelo_ sexuales.pdf Downloaded 17th May 2012.

- Moreno, J. et al. (2008) Importancia de la práctica físico- deportiva y del género en el autoconcepto físico de los 9 a los 23 años. International Journal of Clinical and Health

- Psychology, 8 (1), 171-183. Available: http://www.um.es/ univefd/impoauto.pdf Downloaded 15th May 2012.

- Norman, K. (2007) Valoración de hábitos de alimentación, actividad física y condición nutricional en estudiantes de la pontificia universidad católica de Valparaíso. Rev Chil

- Nutr 34 (4), Available: http://www.scielo.cl/scielo. php?script=sci_arttext\&pid=S0717-75182007000400006. Downloaded 8th May 2012.

- Paglilla, D. (2001) La tercera edad y la actividad física. Revista digital. Buenos Aires. 7, (36) Available: http://www. efdeportes.com/efd36/3aedad.htm. Downloaded 10th May 2012.

- Pérez, S. et al (2007) Estudios sobre alimentación y nutrición en México: una mirada a través del género. Salud pública Méx 49(6), Available: http://www.scielo.org.mx/ scielo.php?pid=S0036-36342007000600012\&script $=$ sci_ arttext Downloaded 8th May 2012.

- Sarabia, C. (2012) La imagen corporal en los ancianos: Estudio descriptivo. Gerokomos 23 (1), 15-18. Available: http://scielo.isciii.es/scielo.php?script=sci_arttext\&pid= S1134-928X2012000100003\&lng=en\&nrm=iso\&tlng=en Downloaded 17th May 2012.

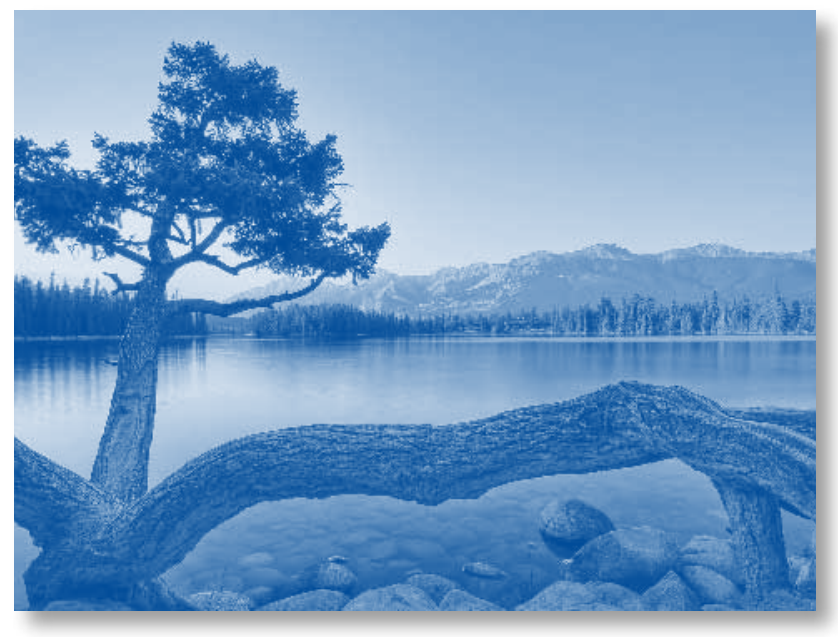

